\title{
ctDNA in Breast Milk for Early Detection of Pregnancy Associated Breast Cancer
}

Cristina Saura ( $\nabla$ csaura@vhio.net )

Vall d'Hebron Institute of Oncology (VHIO)

\section{Carolina Ortiz}

Vall d'Hebron Institute of Oncology

\section{Enrique Arenas Lahuerta}

Vall d'Hebron Institute of Oncology (VHIO) https://orcid.org/0000-0001-7076-2666

\section{Judit Matito}

Vall d'Hebrón Institut d’Oncología (VHIO)

\section{Anna Suñol}

Vall d'Hebron Institute of Oncology

\section{Octavi Córdoba}

Hospital Universitari Son Espases https://orcid.org/0000-0002-9591-7430

\section{Alex Martínez-Sabadell Aliguer}

Vall d'Hebron Institute of Oncology https://orcid.org/0000-0002-9477-1396

Itziar García-Ruiz

Vall d'Hebron Hospital Universitari

Ignacio Miranda

Vall d'Hebron Hospital Universitari https://orcid.org/0000-0002-4813-5946

\section{Clara Morales-Comas}

Vall d'Hebron University Hospital

\section{Estela Carrasco}

Vall d'Hebron Institute of Oncology

\section{Cristina Viaplana}

Vall d’Hebron Institute of Oncology

\section{Vicente Peg}

Universidad Autónoma de Barcelona, Barcelona; Department of Pathology in Vall d'Hebron University Hospital, Barcelona; Spanish Biomedical Research Network Centre in Oncology (CIBERONC)

https://orcid.org/0000-0002-5203-6166

\section{Paolo Nuciforo}

Vall d'Hebron Institute of Oncology https://orcid.org/0000-0003-1380-0990

\section{Neus Bayó-Puxan}

Vall d'Hebron Institute of Oncology

Josep Miquel 
Vall d'Hebron Institute of Oncology (VHIO), Barcelona

\section{Marina Gómez-Rey}

Vall d'Hebron Institute of Oncology (VHIO)

\section{Guillermo Villacampa}

Vall d'Hebron Institute of Oncology (VHIO)

\section{Silvia Arévalo}

Vall d'Hebron Hospital Universitari

\section{Martin Espinosa-Bravo}

Vall d'Hebron Hospital Universitari

\section{Judith Balmaña}

Vall d'Hebron Institut d'Oncologia https://orcid.org/0000-0002-0762-6415

\section{Rodrigo Dienstmann}

all d'Hebron Institute of Oncology, Vall d'Hebron University Hospital, Autonomous University of Barcelona

\section{Joaquín Arribas}

Vall d'Hebron Institute of Oncology https://orcid.org/0000-0002-0504-0664

\section{Josep Tabernero}

Vall d'Hebron Institute of Oncology (VHIO) https://orcid.org/0000-0002-2495-8139

\section{Miriam Sanso ( $\sim$ msanso@vhio.net )}

Vall d'Hebron Institute of Oncology (VHIO)

\section{Ana Vivancos}

Vall d'Hebrón Institut d’Oncología (VHIO)

\section{Brief Communication}

Keywords: ctDNA, breast milk, breast cancer, PABC

Posted Date: August 10th, 2021

DOI: https://doi.org/10.21203/rs.3.rs-757809/v1

License: (c) (i) This work is licensed under a Creative Commons Attribution 4.0 International License. Read Full License 


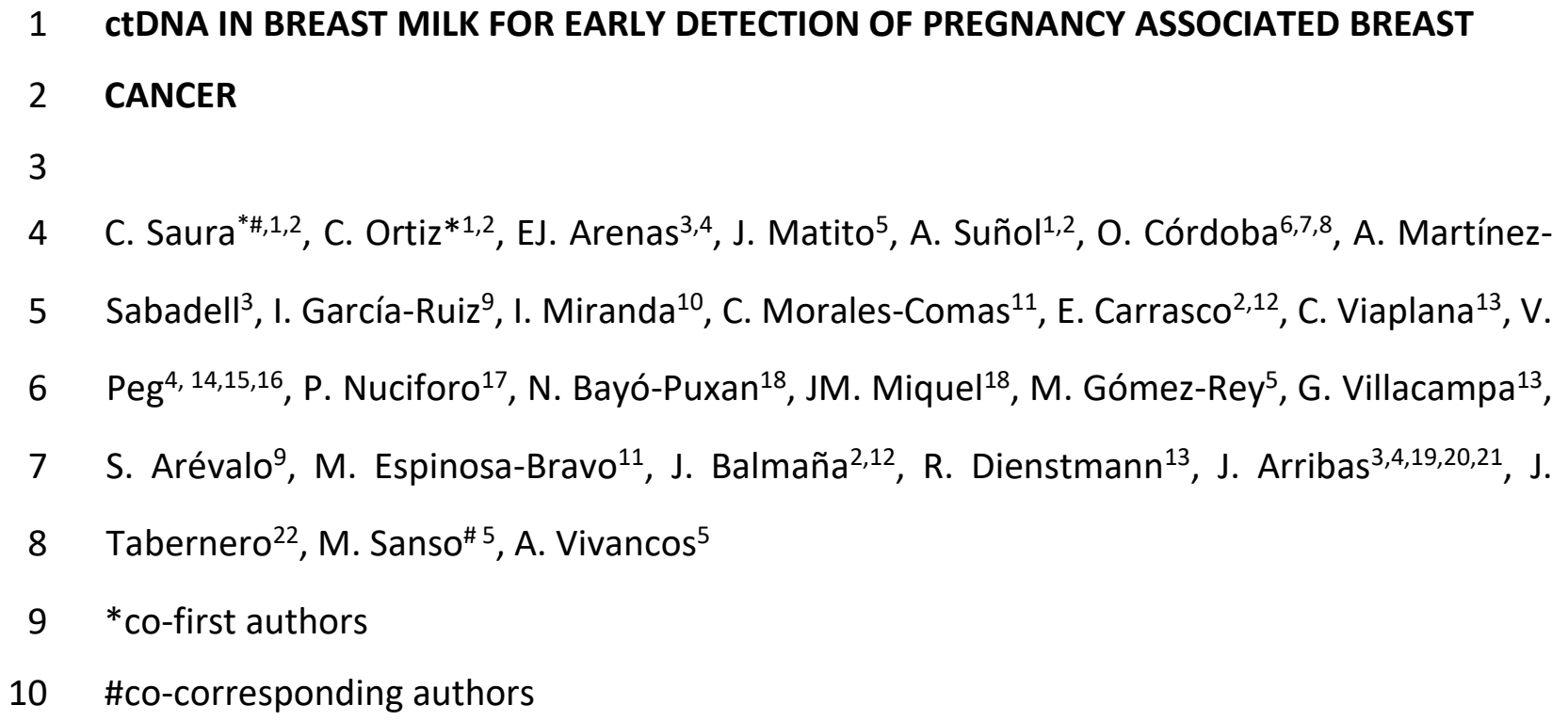

11 1. Breast Cancer and Melanoma Group, Vall d'Hebron Institute of Oncology (VHIO), Vall 12 d'Hebron Barcelona Hospital Campus, C/ Natzaret, 115-117, 08035 Barcelona, Spain

13 2. Medical Oncology Department, Vall d'Hebron University Hospital, Vall d'Hebron 14 Barcelona Hospital Campus, Passeig Vall d'Hebron 119-129, 08035 Barcelona, Spain

15 3. Preclinical \& Translational Research Program, Vall d'Hebron Institute of Oncology 16 (VHIO), Vall d'Hebron Barcelona Hospital Campus, C/ Natzaret, 115-117, 08035 17 Barcelona, Spain

18 4. Centro de Investigación Biomédica en Red en Cáncer (CIBERONC), Instituto de Salud 19 Carlos III, Avenida de Monforte de Lemos 3-5, 28029 Madrid, Spain

20 5. Cancer Genomics Group, Vall d'Hebron Institute of Oncology (VHIO), Vall d'Hebron 21 Barcelona Hospital Campus, C/ Natzaret, 115-117, 08035 Barcelona, Spain

22 6. Servicio Obstetricia y Ginecología, Hospital Universitari Son Espases, Carretera de 23 Valldemosa 79, 07120, Palma, Spain

24 7. IdISBa. Institut de Investigació de les Illes Balears, Carretera de Valldemosa 79, 07120, $25 \quad$ Palma, Spain

26 8. Universitat de les Illes Balears, Carretera de Valldemosa 79, 07120, Palma, Spain 
27 9. Maternal Fetal Medicine Unit, Department of Obstetrics. Vall d'Hebron Hospital 28 Universitari, Vall d'Hebron Barcelona Hospital Campus, Passeig Vall d'Hebron 119-129, 2908035 Barcelona, Spain

30 10. Unitat Diagnòstic per la Imatge de la Dona, Servei de Radiodiagnòstic. Vall d'Hebron 31 Hospital Universitari, Vall d'Hebron Barcelona Hospital Campus, Passeig Vall d'Hebron 32 119-129, 08035 Barcelona, Spain

33 11. Breast Surgical Unit. Breast Cancer Center. Gynecology Department, Hospital 34 Universitari Vall d'Hebron, Vall d'Hebron Barcelona Hospital Campus, Passeig Vall 35 d'Hebron 119-129, 08035 Barcelona, Spain

36 12. Hereditary Cancer Genetics Group, Vall d'Hebron Institute of Oncology (VHIO), Vall 37 d'Hebron Barcelona Hospital Campus, C/ Natzaret, 115-117, 08035 Barcelona, Spain

38 13. Oncology Data Science Group, Vall d'Hebron Institute of Oncology (VHIO), Vall d'Hebron 39 Barcelona Hospital Campus, C/ Natzaret, 115-117, 08035 Barcelona, Spain

14. Servei d'Anatomia Patològica, Vall d'Hebron Hospital Universitari, Vall d'Hebron Barcelona Hospital Campus, Passeig Vall d'Hebron 119-129, 08035 Barcelona, Spain

42 15. Grup de Patologia Molecular, Vall d'Hebron Institut de Recerca (VHIR), Vall d'Hebron 43 Hospital Universitari, Vall d'Hebron Barcelona Hospital Campus, Passeig Vall d'Hebron 119- 129, 08035 Barcelona, Spain

16. Universitat Autònoma de Barcelona, 08193 Bellaterra, Spain

17. Molecular Oncology Group, Vall d'Hebron Institute of Oncology (VHIO), Vall d'Hebron 53 Barcelona Hospital Campus, C/ Natzaret, 115-117, 08035 Barcelona, Spain

18. Scientific Development Area, Vall d'Hebron Institute of Oncology (VHIO), Vall d'Hebron Barcelona Hospital Campus, C/ Natzaret, 115-117, 08035 Barcelona, Spain

19. Department of Biochemistry and Molecular Biology, Universitat Autónoma de Barcelona, Campus de la UAB, 08193, Bellaterra, Spain. Research 
Institute), Barcelona, Spain.

55 21. Institució Catalana de Recerca i Estudis Avançats (ICREA), 08010, Barcelona, Spain.

56

57

58

59

60

61

62

63
22. Vall d'Hebron Hospital Campus and Institute of Oncology (VHIO), UVic-UCC, IOB-Quiron Barcelona, SPAIN

\section{ABSTRACT}

Pregnancy associated breast cancer (PABC) is an overall poor prognosis group compared to non-PABC breast cancer $(B C)$, mainly due to late diagnosis. Effective screening approaches for early detection would likely improve its outcome. For the first time, we demonstrate the presence of cell-free tumor DNA (ctDNA) in the breast milk serum (sBM) of PABC in patients with early-stage disease and its ability to recapitulate somatic mutations present in the primary tumor. Moreover, our results demonstrate that sBM robustly surpasses plasma for tumor genetic profiling due to a prevalent shedding of ctDNA into the SBM in localized disease, as well as increased total cell-free DNA (cfDNA) abundance and integrity. Thus, we propose $S B M$ as a potential new non-invasive liquid biopsy for a prompt detection of PABC.

When $B C$ is diagnosed during pregnancy, lactation, or within one year after delivery, it is known as $P A B C^{1}$. PABC is the most common malignancy and cancer-related death diagnosed during pregnancy and lactating patients, occurring in 1 out of every 3.000 to 10.000 pregnancies ${ }^{1,2}$. The exact incidence is unknown, but considering that $\mathrm{BC}$ risk increases with age and the current trend of delaying pregnancy in developed countries, PABC cases are expected to rise in years to come $\mathrm{e}^{3,4}$.

PABC confers a significantly higher risk of death compared to the non-pregnancy related $B C$, with an even poorer outcome when the diagnosis is postpartum ${ }^{5}$. The main factor behind 
82 the poor prognosis is a delay in diagnosis more than the pregnancy-increased hormonal

83 state $^{6}$. During pregnancy, breast density, volume and water content increase, impairing 84 both clinical and radiologic evaluation ${ }^{1}$. Screening for BC relies mainly in imaging technics

85 focused on postmenopausal women, leaving the population in fertile age with the only 86 option of clinical- and self-examination ${ }^{7}$. Since localized BC detection is critical in reducing 87 mortality, and diagnosis is particularly challenging in pregnant and lactating women, highly 88 sensitive and non-invasive new methods are eagerly awaited for early detection of PABC ${ }^{1}$.

In the era of precision oncology, liquid biopsy arises as a solid candidate for early cancer detection as cfDNA fragments present in the blood or other body fluids may be used as

92 diagnostic biomarkers ${ }^{8}$. Malignant cells release DNA into the bloodstream, known as ctDNA,

93 a small fraction of the total cfDNA and where somatic mutations, methylation and copy number alterations are potentially traceable ${ }^{9}$. Nowadays, ctDNA analysis is well established

95 in the metastatic BC setting, where up to $85 \%$ of the patients release enough ctDNA into 96 the bloodstream for successful detection and its clinical utility has been already defined ${ }^{10}$. 97 As an inflection point of liquid biopsy adoption in BC, the FDA approved the analysis of 98 PIK3CA mutations in both plasma or tissue to select HR+/HER2- advanced BC patients for 99 administration of the PI3K inhibitor alpesilib ${ }^{11}$. ESR1 mutations, a marker of acquired 100 resistance to endocrine therapies, are also robustly identified in plasma and considered for 101 clinical decisions ${ }^{12}$. In contrast, localized tumors release less ctDNA into the blood, 102 challenging its detection with current tecniques ${ }^{13}$. This obstacle is especially relevant in 103 early BC, where only up to 35 to $50 \%$ of the patients present detectable ctDNA in plasma ${ }^{14,15}$ 104 and this may dramatically drop to only $16 \%$ in stages I and $\mathrm{II}^{16}$.

105

106 Breast milk (BM) originates from the lobular tissue distributed throughout the mammary 107 gland and mammary ducts carry the milk from the lobules during breastfeeding. Upon 108 maturation, the Erk1/2 pathway regulates an apical/basal polarization of the mammary 109 epithelial cells to ensure directional secretion of milk into the lumen of the mammary gland. 
110 Tight junction protein complexes build a blood-milk barrier that partly isolates the breast

111 microenvironment from systemic circulation ${ }^{17}$. BM has proven to be a non-invasive source

112 for studying mature gland cellularity and its microenvironment ${ }^{18}$, genomic promoter

113 methylation of specific $\mathrm{CpGs}^{19}$, cancer-associated miRNAs ${ }^{20}$, cancer stem-like cells ${ }^{21}$ or

114 dysregulation of proteins as possible biomarkers of BC risk ${ }^{22}$. These previous findings, the

115 physical properties of the mammary gland, its partial isolation from the systemic circulation

116 and the intimate contact with the tumor, led us to hypothesize that BM might contain

117 detectable ctDNA, probably before than in blood-derived plasma.

119 To test this hypothesis, during a period of 36 months we enrolled 16 PABC patients 120 diagnosed at different stages of the disease who had available tumor tissue (Table 1 and 121 Supp. Table 1). DNA obtained from FFPE-derived tissues was analyzed by NGS with a custom 122 432-gene hybrid capture-based panel (VHIO-300 panel, Supp. Table 2) in all cases except for 123 patient 1, whose primary tissue was analyzed by NGS with a similar panel after enrollment 124 in the AURORA (NCT02102165) clinical trial (411 genes, lon torrent, see Supp. Table 3) ${ }^{23}$. 125 Genetically, all tumors showed single nucleotide variants, frameshifts and/or copy number 126 alterations (Figure 1A-B and Supp. Tables 4, 5). Consistently with previous observations, a 127 high prevalence of mutations in GATA3 was observed, probably due to the young median 128 age (36 years, range 25-42) and high proportion of luminal tumors of the patient cohort $129(81 \%)^{24}$ (Table 1), followed by PIK3CA, TP53 and KMT2C. The data was also analyzed for 130 homologous recombination deficiency status (HRD). 43,75\% of the tumors where HRD-high 131 according to Marquard et al. ${ }^{25} \mathrm{HRD}$ scoring $\geq 42$ (Figure $1 \mathrm{~A}$ and Supp Table 1 ). This result 132 correlates with a previous study in an European cohort of 70 BC samples from young 133 patients, with high representation of TNBC tumors ${ }^{26}$, a known subtype for its enrichment in 134 HR pathway deficiency ${ }^{27}$. Interestingly, our set of cases is biased towards luminal PABC 135 tumors and still with high percentage of HR defective phenotype.

137 Out of the 16 patients enrolled in the study, 4 cases were excluded for further analysis. 138 Briefly, 3 cases were discarded due to tumor surgery before childbirth (patients 13, 15 and 
139 16), and in 1 case there was no production of BM, probably due to neoadjuvant 140 chemotherapy treatment (case 14) (Supp. Table 1). The remaining 12 cases, with available 141 BM sample, were considered for further analysis, although the first two had no synchronous 142 plasma collected (Supp. Figure 1). In order to study the presence of ctDNA in BM, we 143 fractionated the sample by centrifugation to isolate the cellular fraction and the milk fat, 144 analyzing only the sBM. We chose to test the presence of ctDNA in SBM with ddPCR, the 145 most sensitive and quantitative technique available at that time. Custom assays were 146 successfully validated in the FFPE-derived DNA (Figure 1C, Supp. Table 1). Strikingly, 10 out 147 of 12 of the sBM samples were positive for the presence of the mutant alleles matching the 148 tumor tissues. To assess whether sBM is a superior source of ctDNA than plasma, we 149 performed the same analysis in the plasma samples synchronously collected with the BM. 150 Only 1 out of 10 samples presented ctDNA, in a patient diagnosed with locally advanced BC 151 in stage IIIC, the highest stage in the cohort (Supp. Table 1). This result is in agreement with 152 the lower rates of plasma ctDNA in localized $\mathrm{BC}^{10}$. But more importantly, it confirms 153 expeditious detectability and superior sensitivity of ctDNA in sBM versus plasma, even in a 154 patient with a stage IA tumor.

156 Interestingly, relative abundance of ctDNA in SBM did not appear to correlate with disease 157 (tumor, nodal status nor stage), in a similar fashion to previous reports analyzing blood158 derived plasma (Figure 1D). Importantly, the two samples that resulted negative for 159 mutated alleles in sBM were isolated from colostrum, collected the first day after delivery. 160 These result lead us to speculate whether maturity of the milk affects the sensitivity of the 161 assay. Thus, we classified all samples as colostrum, collected between day 1 and 5 after 162 delivery; transitional, collected from day 6 to 15 of lactation; and mature, after day 16 until 163 the end of lactation (Supp. Table 1$)^{28}$. We split immature milk samples, including colostrum 164 and transitional, from mature ones, and observed that the milk collection day from delivery 165 is crucial for increasing ctDNA detection, since the lowest MAFs were observed in immature 166 milk specimens in a statistically significant manner (Figure 1D). Therefore, we hypothesize 167 that restricting collection of samples to mature BM specimens might increase sensitivity of 
168 the protocol to a $100 \%$ (in fact, the actual sensitivity of samples classified as

169 transitional/mature collected in our project). Other factors that correlated with sBM MAF

170 detection were the molecular subtype (luminal A versus luminal B), and the diagnosis of an

171 invasive lobular carcinoma (ILC) versus a ductal one (Figure 1D). The last observation may

172 be explained due to the origin of ILC in the milk-producing glands of the breast, where cells

173 experience a higher pressure for milk extraction compared to the cells form the ducts, which

174 simply transport the BM. Moreover, ILC is characterized by small non-cohesive neoplastic

175 cells invading the stroma due to dysregulation of cell-cell adhesion, primarily driven by lack

176 of E-cadherin $(\mathrm{CDH} 1)^{26}$. Indeed, both ILC cases in our cohort had a truncating mutation in

$177 C D H 1$, classified as likely pathogenic (Figure $1 \mathrm{~A}$ and Supp.Table 4). Such loss of tightness

178 between cells might also influence the higher DNA shedding capacity of ILC subtype into the

179 sBM.

180

181 One of the most exciting results came from case 1, whose milk sample was not synchronous 182 to BC diagnosis. Instead, the patient was diagnosed during her third pregnancy, and 183 provided a sample from the previous lactation of her second child. Thus, the sample was 184 collected 18 months before diagnosis, but still a 4,05\% of PIK3CA alleles harbored the 185 p.H1047R mutation detected in the tumor (Figure 1C and Supp. Table 1). This result, along 186 with a high sensitivity for ctDNA detection reported, strongly makes us postulate that sBM 187 genomic profiling may be a powerful tool for early detection of PABC.

188 Interestingly, case 4, corresponding to a patient with a luminal A stage IA tumor showed a $18915 \%$ of $C B F B$ p.W113X allelic frequency and $1460 \mathrm{ng} / \mathrm{ml}$ of cfDNA in sBM, showing the 190 second highest detected MAF and also one of the highest DNA concentrations in the cohort 191 (and Supp. Figure 2A and Supp. Table 1). A plausible explanation for this interesting result 192 is not only its lobular tumor origin but also that the patient was suppressing breastfeeding 193 of a previous child at the time of BC diagnosis and sBM collection coincided with the 194 involution of the mammary gland, a stage where a dramatic remodeling and intensive 195 cellular apoptosis occurs ${ }^{29}$. 
197 A main limitation of liquid biopsy is the scarce concentration of cfDNA and high dilution of 198 ctDNA. Even though sBM volumes varied considerably among patients, we obtained on 199 average $486,5 \mathrm{ng}$ of cfDNA from each $\mathrm{ml}$ of sBM. This was approximately 100 -fold that of 200 blood, with a median of 5,7 ng of cfDNA obtained per $\mathrm{ml}$ ( $\mathrm{p}$-val $<0.0001$ ) in the PABC cohort 201 of patients, or $5,2 \mathrm{ng} / \mathrm{ml}$ obtained from 56 metastatic BC plasma samples previously 202 analyzed in our laboratory (Supp. Figure 2A). Therefore, the number of genome equivalents 203 available for analysis in the sBM is also superior than in plasma, a very convenient feature 204 for an early detection screening approach.

206 Another trait of cfDNA is its pattern of fragmentation ${ }^{30}$. To study the fragmentome of sBM 207 cfDNA we performed lab-on-a-chip assay on the samples from each patient, and compared 208 to healthy controls of plasma and sBM (Supp. Figure 2B). For patients 1 and 2 we used 209 asynchronous plasma samples collected during response to treatment. Importantly, most 210 sBM samples presented a wide length distribution of DNA molecules, ranging from 100 until 211 far beyond $10380 \mathrm{bp}$ (the upper limit of resolution). The expected peak of $167 \mathrm{bp}$ 212 corresponding to mononucleosomal DNA, a common feature in plasma samples, was not 213 always prominent or the major size peak (Supp. Figure 2B right panel). Finally, no significant 214 difference in amount of cfDNA nor in fragmentome was observed between the affected and 215 the healthy breast, when both samples were available (data not shown) or when compared 216 to a healthy control sBM sample (Supp. Figure 2B right panel). More detailed studies are 217 required to understand the nature of the SBM cfDNA fragmentome. For instance, it would 218 be interesting to clarify whether a different mixture of nucleases present in the milk or 219 composition of extracellular vesicles protecting the DNA are responsible for the observed 220 pattern, but the present work clearly revealed a more variable fragmentome with a 221 generalized higher DNA integrity compared to plasmatic cfDNA. Indeed, contrary to blood222 derived plasma, it has been reported that the fat layer of the BM is a good source of RNA to 223 profile gene expression of the breast epithelium, due to the protection of the molecules 224 inside of milk fat globules ${ }^{31}$. 
226 The number of cases in this study is limited, mainly due to the difficulty to recruit PABC 227 patients in the clinic with synchronous collection of BM and blood samples. That being said, 228 the results among the cohort are quite compelling and demonstrate for the first time: I) the 229 presence of ctDNA in 83,33\% of the sBM samples from patients diagnosed with PABC $(100 \%$ 230 if only transitional and mature milk samples considered). II) ctDNA in SBM may be detected 231 in localized disease even in stage IA. III) ctDNA is detectable in sBM prior to plasma in 91\% 232 of the synchronous analyzed samples. IV) ctDNA shedding in BM could anticipate PABC 233 diagnosis, according to results from case 1. V) Maturation of the milk sample is crucial for 234 the sensitivity of the sBM genomic analysis of ctDNA. VI) Sensitivity of the sBM as liquid 235 biopsy might be higher in ILC tumor type since it liberates more ctDNA into the sBM than 236 IDC. VII) cfDNA concentration in SBM is 100 times higher than in blood. VIII) cfDNA integrity 237 from BM is superior when compared to cfDNA from plasma.

To our knowledge this is the first study reporting the presence of ctDNA in BM. Moreover, 240 in the light of the results obtained from sBM demonstrating the higher sensitivity, cfDNA 241 concentration and fragmentome integrity compared to blood-derived plasma, we propose 242 BM genomic analysis as the most appropriate approach for PABC early detection. This could 243 be the first specific PABC early detection method for a population that lacks of any other 244 opportunity for early screening of BC, were stablished protocols are generally based on 245 imaging and performed exclusively in postmenopausal women.

\section{REFERENCES} Amant F, Loibl S, Neven P, Van Calsteren K. Malignancies in Pregnancy 2 Breast cancer in

252 2. Maxwell C V., Al-Sehli H, Parrish J, D’Souza R. Breast Cancer in Pregnancy: A Retrospective 253 Cohort Study. Gynecol Obstet Invest. 2019;84(1):79-85. doi:10.1159/000493128 
254 3. Polyak K. Pregnancy and breast cancer: The other side of the coin. Cancer Cell. 2006;9(3):151-

255 153. doi:10.1016/j.ccr.2006.02.026

256 4. Ruiz R, Herrero C, Strasser-Weippl K, et al. Epidemiology and pathophysiology of pregnancy-

257 associated breast cancer: A review. Breast. 2017;35:136-141.

258 doi:10.1016/j.breast.2017.07.008

259 5. Ulery M, Carter L, McFarlin BL, Giurgescu C. Pregnancy-Associated Breast Cancer:

260 Significance of Early Detection. J Midwifery Womens Health. 2009;54(5):357-363.

261 doi:10.1016/j.jmwh.2008.12.007

262 6. Amant F, Von Minckwitz G, Han SN, et al. Prognosis of women with primary breast cancer

263 diagnosed during pregnancy: Results from an international collaborative study. J Clin Oncol.

264 2013;31(20):2532-2539. doi:10.1200/JCO.2012.45.6335

265 7. Pashayan N, Antoniou AC, Ivanus U, et al. Personalized early detection and prevention of

266 breast cancer: ENVISION consensus statement. Nat Rev Clin Oncol. 2020;17(11):687-705.

267 doi:10.1038/s41571-020-0388-9

268 8. Rohanizadegan M. Analysis of circulating tumor DNA in breast cancer as a diagnostic and prognostic biomarker. Cancer Genet. 2018;228-229:159-168. doi:10.1016/j.cancergen.2018.02.002

271 9. Rennstam K, Ahlstedt-Soini M, Baldetorp B, et al. Patterns of chromosomal imbalances 272 defines subgroups of breast cancer with distinct clinical features and prognosis. A study of 305 tumors by comparative genomic hybridization. Cancer Res. 2003;63(24):8861-8868. http://www.ncbi.nlm.nih.gov/pubmed/14695203. Accessed February 17, 2020.

275 10. Bettegowda C, Sausen M, Leary RJ, et al. Detection of circulating tumor DNA in early- and 276 late-stage human malignancies. Sci Transl Med. 2014;6(224). doi:10.1126/scitranslmed.3007094

278 11. André F, Ciruelos E, Rubovszky G, et al. Alpelisib for PIK3CA -Mutated, Hormone ReceptorPositive Advanced Breast Cancer . N Engl J Med. 2019;380(20):1929-1940. doi:10.1056/nejmoa1813904

281 12. O'Leary B, Hrebien S, Morden JP, et al. Early circulating tumor DNA dynamics and clonal selection with palbociclib and fulvestrant for breast cancer. Nat Commun. 2018;9(1):1-10. doi:10.1038/s41467-018-03215-x

284 13. Phallen J, Sausen M, Adleff V, et al. Direct detection of early-stage cancers using circulating 
tumor DNA. Sci Transl Med. 2017;9(403). doi:10.1126/scitranslmed.aan2415

14. Cohen JD, Li L, Wang Y, et al. Detection and localization of surgically resectable cancers with a multi-analyte blood test. Science (80- ). 2018;359(6378):926-930. doi:10.1126/science.aar3247

15. Mattox AK, Bettegowda C, Zhou S, Papadopoulos N, Kinzler KW, Vogelstein B. Applications of liquid biopsies for cancer. Sci Transl Med. 2019;11(507):1984. doi:10.1126/scitransImed.aay1984

16. Yoshinami T, Kagara N, Motooka D, et al. Detection of ctDNA with Personalized Molecular Barcode NGS and Its Clinical Significance in Patients with Early Breast Cancer. Trans/ Oncol. 2020;13(8). doi:10.1016/j.tranon.2020.100787

17. Liu F, Pawliwec A, Feng Z, Yasruel Z, Lebrun JJ, Ali S. Prolactin/Jak2 directs apical/basal polarization and luminal linage maturation of mammary epithelial cells through regulation of the Erk1/2 pathway. Stem Cell Res. 2015;15(2):376-383. doi:10.1016/j.scr.2015.08.001

19. Wong CM, Anderton DL, Smith-Schneider S, Wing MA, Greven MC, Arcaro KF. Quantitative

20. Qin W, Tsukasaki Y, Dasgupta S, Mukhopadhyay N, Ikebe M, Sauter ER. Exosomes in human breast milk promote EMT. Clin Cancer Res. 2016;22(17):4517-4524. doi:10.1158/10780432.CCR-16-0135

21. Bhat-Nakshatri P, Kumar B, Simpson E, et al. Breast Cancer Cell Detection and

22. Aslebagh R, Channaveerappa D, Arcaro KF, Darie CC. Proteomics analysis of human breast milk to assess breast cancer risk. Electrophoresis. 2018;39(4):653-665. doi:10.1002/elps.201700123

23. Maetens $M$, Brown D, Irrthum A, et al. The AURORA pilot study for molecular screening of patients with advanced breast cancer-a study of the breast international group. npj Breast Cancer. 2017;3(1). doi:10.1038/s41523-017-0026-6 
316 24. Azim HA, Brohée S, Peccatori FA, et al. Biology of breast cancer during pregnancy using genomic profiling. Endocr Relat Cancer. 2014;21(4):545-554. doi:10.1530/ERC-14-0111

318 25. Marquard AM, Eklund AC, Joshi T, et al. Pan-cancer analysis of genomic scar signatures associated with homologous recombination deficiency suggests novel indications for existing cancer drugs. Biomark Res. 2015;3(1):1-10. doi:10.1186/s40364-015-0033-4

321 26. Watanabe T, Honda T, Totsuka H, et al. Simple prediction model for homologous recombination deficiency in breast cancers in adolescents and young adults. Breast Cancer Res Treat. 2020;182(2):491-502. doi:10.1007/s10549-020-05716-0

324 27. Timms KM, Abkevich V, Hughes $E$, et al. Association of BRCA1/2 defects with genomic scores predictive of DNA damage repair deficiency among breast cancer subtypes. Breast Cancer Res. 2014;16(1). doi:10.1186/s13058-014-0475-x

28. Neville MC. Anatomy and physiology of lactation. Pediatr Clin North Am. 2001;48(1):13-34. doi:10.1016/S0031-3955(05)70283-2

29. Jena MK, Jaswal S, Kumar S, Mohanty AK. Molecular mechanism of mammary gland involution: An update. Dev Biol. 2019;445(2):145-155. doi:10.1016/j.ydbio.2018.11.002

30. Cristiano S, Leal A, Phallen J, et al. Genome-wide cell-free DNA fragmentation in patients with cancer. Nature. 2019;570(7761):385-389. doi:10.1038/s41586-019-1272-6

31. Maningat PD, Sen P, Rijnkels M, et al. Gene expression in the human mammary epithelium during lactation: the milk fat globule transcriptome. Physiol Genomics. 2009;37(1):12-22. doi:10.1152/physiolgenomics.90341.200

338 We would like to thank the generous contribution of the patients who participated in the 339 study and donated samples for analysis, with particular thanks to the first patient, Maite, 340 and her daughter Àneu, who inspired us to initiate this study. Our team also gratefully 341 acknowledges the funding support of the patient's association "El paseíco de la mama" that 342 unconditionally supported our research in PABC, as well as the FERO Foundation for its 343 support of research at the Vall d'Hebron Institute of Oncology (VHIO), in particular for the 344 "II FERO-GHD grant" and the DIAMAV program. We also acknowledge the 
345 Spanish Association Against Cancer Foundation (FAECC) for funding Cancer Genomics Lab

346 research and for funding Miriam Sansó's research work with an AECC Investigator award.

347 Finally, we acknowledge CERCA Programme/Generalitat de Catalunya for Institutional

348 support, the CELLEX Foundation for providing research facilities and equipment.

\section{DISCLOSURE OF POTENTIAL CONFLICTS OF INTEREST}

350 C. Saura have served as consultant, participated in advisory boards or received travel grants from

351 AstraZeneca, Celgene, Daiichi Sankyo, Eisai, Roche, Genomic Health, MSD, Novartis, Pfizer, Philips

352 Healthwork, Pierre Fabre, prIME Oncology, Puma Biotechnology, Synthon and Sanofi Aventis.

353

354 O. Córdoba was member of a scientific advisory board at GE and Ascires sistemas genómicos and

$355 \mathrm{GE}$, reports receiving commercial educational grants from Roche diagnostics, 356 Neomedic and Takeda.

357 V. Peg have Received grants from Roche, played and advisory role for Roche, MSD, AstraZeneca and 358 have received honoraria from Roche and Sysmex Spain.

359 P. Nuciforo has consulted for Bayer, Novartis, MSD, and Targos, and received compensation.

360 R. Dienstmann has declared advisory role for Roche, Boehringer Ingelheim, has received a speaker's

361 fee from Roche, Ipsen, Amgen, Servier, Sanofi, Merck Sharp \& Dohme and Medscape, and research 362 grants from Merck and Pierre Fabre.

363 J. Arribas has received research funds from Roche, Synthon, Menarini, and Molecular Partners and 364 consultancy honoraria from Menarini.

365 J. Tabernero reports personal financial interest in form of scientific consultancy role for Array 366 Biopharma, AstraZeneca, Avvinity, Bayer, Boehringer Ingelheim, Chugai, Daiichi Sankyo, F. 367 Hoffmann-La Roche Ltd, Genentech Inc, HalioDX SAS, Hutchison MediPharma International, Ikena 368 Oncology, IQVIA, Lilly, Menarini, Merck Serono, Merus, MSD, Mirati, Neophore, Novartis, Orion 369 Biotechnology, Peptomyc, Pfizer, Pierre Fabre, Samsung Bioepis, Sanofi, Seattle Genetics, Servier, 370 Taiho, Tessa Therapeutics and TheraMyc. And also, educational collaboration with Imedex, 
371 Medscape Education, MJH Life Sciences, PeerView Institute for Medical Education and Physicians 372 Education Resource (PER).

373 A. Vivancos is a scientific advisory board member at Incyte, Novartis, Merck, Roche, Bristol-Meyers374 Squibb, Guardant Health and Bayer, consultant at Sysmex, reports receiving preclinical research 375 grants from Bristol-Meyers-Squibb, Novartis and Roche, and has received Intellectual Property 376 royalties (Technology Transfer DX Fields) from Ferrer.

377 No potential conflicts of interest were disclosed by the other authors.

\section{AUTHORSHIP}

379 Conception and design of the work: CS, CO, EA, OC, NB, JM, RD, JA, JT, MS, AV. Development of 380 methodology: CS, CO, EA, JM, AMS, JA, MS, AV. Acquisition of data (acquired and managed patients, 381 provided facilities, etc.): CS, CO, EA, JM, AS, AMS, IG, IM, CM, EC, VP, PN, SA, ME, JB, MS, AV. Analysis 382 and interpretation of data (e.g., statistical analysis, biostatistics, computational analysis): CS, CO, EA, $383 \mathrm{JM}, \mathrm{MG}, \mathrm{GV}, \mathrm{AMS}, \mathrm{CV}, \mathrm{RD}, \mathrm{JA}, \mathrm{JT}, \mathrm{MS}, \mathrm{AV}$. Writing, review, and/or revision of the manuscript: All 384 authors. Administrative, technical, or material support (i.e., reporting or organizing data, creation 385 of databases): CO, EA, AS, CV, NB, JM, RD. Study supervision: CS, CO, JT, MS, AV. 


\section{$395 \quad$ Figures and Tables}

\begin{tabular}{|c|c|c|c|}
\hline & & Total & $\%$ \\
\hline$N$ & & 16 & 100 \\
\hline Age & Median (range) & 36 years & $(25-42)$ \\
\hline \multirow{4}{*}{$p T$} & 1 & 4 & $25 \%$ \\
\hline & 2 & 7 & $44 \%$ \\
\hline & 3 & 4 & $25 \%$ \\
\hline & 4 & 1 & $6 \%$ \\
\hline \multirow{2}{*}{$p N$} & 0 & 7 & $44 \%$ \\
\hline & $\geq 1$ & 9 & $56 \%$ \\
\hline \multirow{2}{*}{$M$} & 0 & 15 & $94 \%$ \\
\hline & 1 & 1 & $6 \%$ \\
\hline \multirow{4}{*}{ Stage } & I & 1 & $6 \%$ \\
\hline & II & 9 & $56 \%$ \\
\hline & III & 5 & $31 \%$ \\
\hline & IV & 1 & $6 \%$ \\
\hline \multirow{2}{*}{$H R$} & Positive & 14 & $88 \%$ \\
\hline & Negative & 2 & $13 \%$ \\
\hline \multirow{2}{*}{ HER2 } & Positive & 1 & $6 \%$ \\
\hline & Negative & 15 & $94 \%$ \\
\hline \multirow{4}{*}{ Subtype* } & Luminal $A$ & 4 & $25 \%$ \\
\hline & Luminal B & 9 & $56 \%$ \\
\hline & HER2+ & 1 & $6 \%$ \\
\hline & $T N B C$ & 2 & $13 \%$ \\
\hline \multirow{4}{*}{ Diagnosis } & Trimester 1 & 3 & $19 \%$ \\
\hline & Trimester 2 & 3 & $19 \%$ \\
\hline & Trimester 3 & 4 & $25 \%$ \\
\hline & Lactancy & 6 & $38 \%$ \\
\hline \multicolumn{4}{|c|}{ *Subtype based on Immunohistochemistry. } \\
\hline \multicolumn{4}{|c|}{ Luminal A is defined as ER or PR positive, HER2 negative and Ki67 $<20 \%$} \\
\hline \multicolumn{4}{|c|}{ Luminal $B$ is defined as ER or PR positive, HER2 negative and Ki67 $>=20 \%$} \\
\hline \multicolumn{4}{|c|}{ HER2+ is defined as HER2 positive, regardless ER, PR and Ki67 status } \\
\hline \multicolumn{4}{|c|}{ TNBC is defined as ER, PR and HER2 negative } \\
\hline
\end{tabular}

397 Table 1. Clinical-pathological characteristics of the cohort. 
A

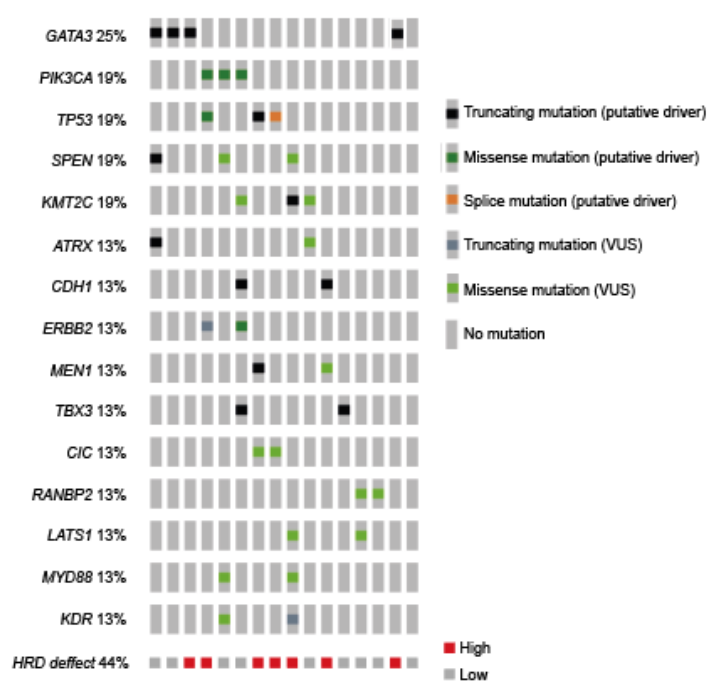

B

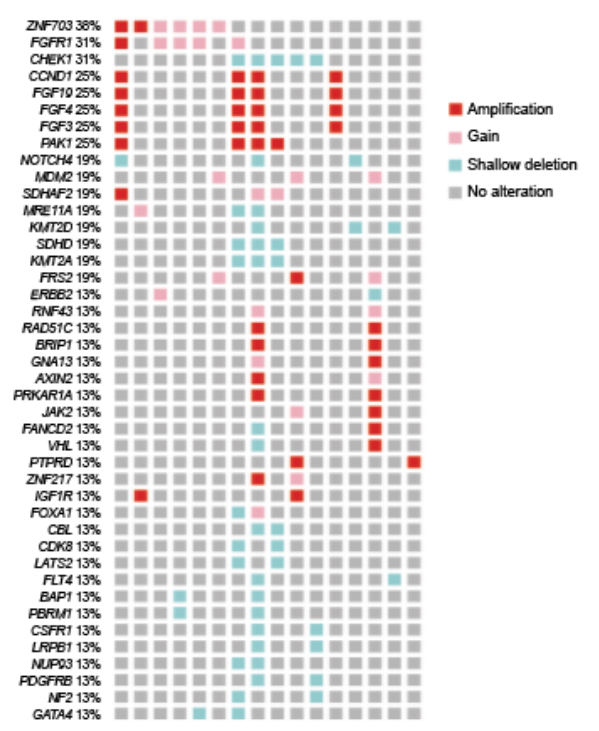

\section{C}

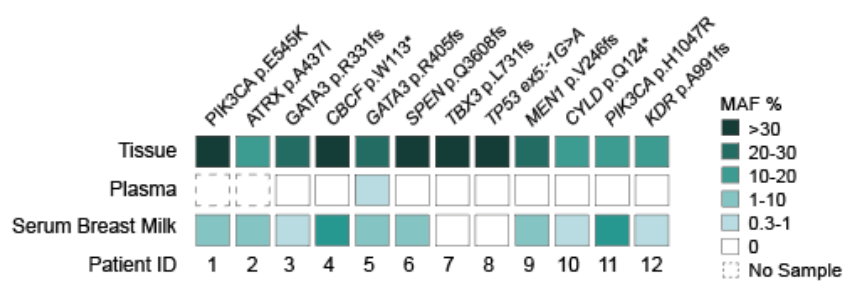

D
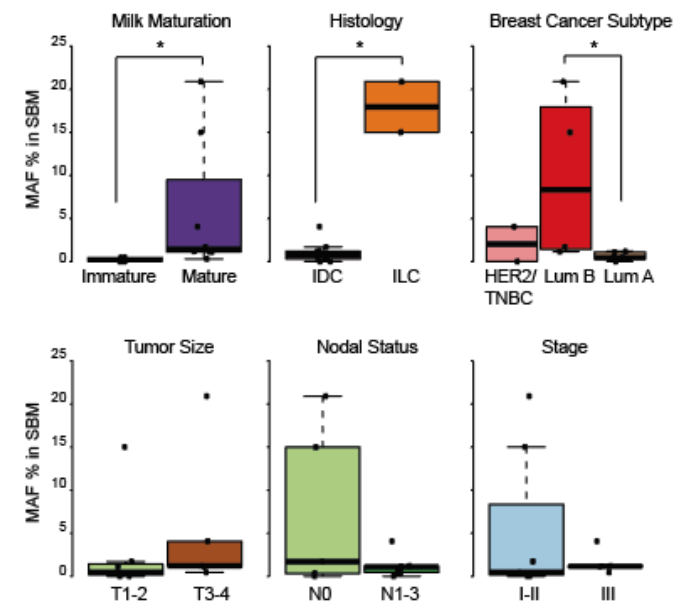

Figure 1. A. Oncoprint of somatic mutations detected by NGS in PABC tumors at least in 2 samples out of 16, and HRD score defined as high when $\geq 42$.B. Oncoprint of CNA alterations detected by NGS in PABC tumors occurring at least in 2 samples out of 16. C.MAF \% detected by ddPCR in solid tissue, plasma and sBM samples, all performed for the most abundant somatic mutation detected in the solid tissue by previous NGS. D. Statistical significance and correlations between MAF \% and milk maturation, histology, breast cancer subtype, nodal status, disease stage, and tumor size. Mann-Whitney Wilcoxon t-test analysis was performed for the different features (* depicts statistical significance with $p$-val < 0.05). 


\section{Supplementary Files}

This is a list of supplementary files associated with this preprint. Click to download.

- SauraNatMedSupplementalfiguresandtables.docx 